\title{
Glaucoma: Risk Factors and Prevalence: A Review
}

\author{
Shaurya Sharma ${ }^{1}$, Harpriya Singh ${ }^{2}$ \\ ${ }^{1}$ MD Ophthalmology, Ex-Regional Institute Of Ophthalmology, Amritsar, ${ }^{2}$ MBBS, GMC Amritsar.
}

\begin{abstract}
In recent time, the concept and definitions of glaucoma have changed from a single pathologic entity to a group of disorders with different clinical pictures. Glaucoma can be defined as a multifactorial optic neuropathy which may or may not be associated with angle abnormality in the presence or absence of any cause for the disease. Irrespective of the manifestation; glaucoma is the second leading cause of blindness worldwide, with preponderance in females, blacks and Asians. Various classifications have been proposed from time to time with the most basic classification system involves separation of angle-closure glaucoma from open-angle glaucoma. This review highlights the clinical features, classification and recent factors related to glaucoma.
\end{abstract}

Key Words: Aqueous humour; Glaucoma; Intraocular; Pressure; Classification

Corresponding author: Shaurya Sharma, MD Ophthalmology, Ex-Regional Institute Of Ophthalmology, Amritsar. Email: shauryasharma.eye @ gmail.com

This article may be cited as: Sharma S and Singh H . Glaucoma: Risk Factors and Prevalence: A Review. Int J Com Health and Med Res. 2016;2(2):66-72

\section{Article Received: 21-05-16}

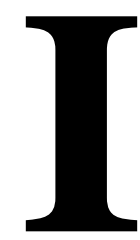

\section{NTRODUCTION}

The concept and definitions of glaucoma have evolved over recent times from a single disease entity to a group of disorders different in their clinical profile, pathophysiology and management. ${ }^{1}$

Glaucoma can be defined as a multifactorial optic neuropathy ${ }^{2}$ with a characteristic accelerated degeneration of retinal ganglion ${ }^{3}$ cells presenting as classical optic nerve head features ${ }^{4}$ and correlating visual field changes, which may or may not be associated with angle abnormality in the presence or absence of any cause for the disease. ${ }^{5,6}$ These disorders share features of cupping and atrophy of the optic nerve head, with attendant visual field loss and are frequently (but not always) related to the level of intraocular pressure(IOP). ${ }^{1}$ Because IOP presently the only factor that can be controlled to prevent progression of optic neuropathy, aqueous humour dynamics, closely related to ocular pressure are critical to glaucoma

\section{Accepted On: 05-06-2016}

management. $^{7,8}$ IOP is determined by the equilibrium between rate of aqueous humour production by ciliary body, the resistance to aqueous outflow at the angle of the anterior chamber (AC), and the level of episcleral venous pressure. The resistance to damage of the optic nerve axons determines the optic nerve head and visual field changes. ${ }^{1}$ current classifications of glaucoma are based on the initial events that in due course, lead to elevated IOP or the alterations in aqueous humour dynamics that are directly responsible for the increase. Continuous research, (including genetics) about the various risk factors lead to the ever changing concepts of glaucoma. However, three crucial parameters- IOP, the optic nerve, and the visual field are the core to our current understanding. ${ }^{9}$ classically, primary glaucomas do not have an association with known ocular or systemic disorders to account for the increased resistance to the aqueous outflow. ${ }^{10}$ on the other hand, the secondary glaucomas are 
associated with ocular or systemic abnormalities responsible for elevated IOP. This dissection into primary and secondary reflects our incomplete understanding of the pathophysiology of the common final pathway of the glaucomatous optic nerve head damage and visual field loss. ${ }^{11}$ Irrespective of the manifestation; glaucoma is the second leading cause of blindness worldwide, with preponderance in females, blacks and asians. ${ }^{12-15}$

\section{CLASSIFICATION}

The most basic classification system is the separation of angle-closure glaucoma from openangle glaucoma. Moving beyond the historical definition of angle-closure glaucoma, the presentday proposal emphasizes on the ultimate pathogenic pathway mechanism of irido-trabecular impediment which results in functional angle closure. ${ }^{16}$ The current classification scheme is a mix of both the natural history of disease depicting the progressive stages, and a mechanistic scheme describing the sites of dysfunction. Clinicoetiological classification ${ }^{17}$ :

I. Open Angle Glaucoma: In open-angle glaucoma, there is relative impairment of flow of aqueous humour through the trabecular meshwork (TM)-schlemm's canal-venous system with an open angle and normal-looking AC.

II. Angle Closure Glaucoma: In angle closure glaucoma resistance to outflow is increased because peripheral iris obstructs the TM.

III. Combined Mechanism Glaucoma: Combination of two or more forms of glaucoma, sequentially or coincidentally.

IV. Childhood Glaucomas: In childhood or developmental glaucomas, anterior segment dysgenesis either presents at birth or appear in the first decades of life.

Globally, primary open-angle glaucoma affects more people than angle-closure with an approximate ratio of $3: 1$, with wide variations among populations and an almost reversal of the global trend in south Asia. However, the symptomatology of angle-closure glaucoma warrants more clinical consult by patients than patients with the chronic benign open-angle glaucoma. ${ }^{14,18}$ Classification Based On Pathogenesis And Treatment: ${ }^{1}$

Classification of open angle glaucoma:
1) Primary open angle glaucoma

2) Normal tension glaucoma

3) Secondary open angle glaucoma

a. Increased resistance to tm outflow associated with other conditions:

(i) pigment dispersion

(ii) pseudoexfoliation

(iii) phacolytic (lens induced) glaucoma

(iv) steroids

b. Increased resistance posterior to tm secondary to elevated episcleral venous pressure (e.g. Carotid cavernous sinus fistula, superior vena cava obstruction, sturge-weber syndrome).

c. Iatrogenic

(i) Glaucoma after cataract surgery

(ii) Glaucoma after vitrectomy (intraocular gas/silicone oil)

d. Glaucoma after trauma (chemical burns, electric shock, radiation, penetrating injury, contusion injury)

e. Glaucoma associated with intraocular haemorrhage

f. Glaucoma associated with retinal detachment

i. Glaucoma associated with uveitis (e.g. Fuchs's heterochromic iridocyclitis, trabeculitis, herpes simplex and zoster, sarcoidosis, juvenile rheumatoid arthritis, syphilis, and hiv).

j. Glaucoma with intraocular tumours

Classification of Angle Closure Glaucoma:

\section{Primary angle closure disease}

Irido-trabecular apposition obstructs aqueous outflow as the final pathology.

a) Natural history

i. Primary angle closure suspect

ii. Primary angle closure

iii. Primary angle-closure glaucoma

b) Anterior segment mechanisms of closure

i. Iris-pupil obstruction(e.g. 'Pupillary block')

ii. Ciliary body anomalies(e.g. 'plateau iris syndrome')

iii. Lens-pupil block (e.g. 'phacomorphic block')

\section{Secondary angle closure}

a) Anterior 'pulling mechanism'

The iris is pulled forward by angle pathology, such as the contraction of a 
membrane or peripheral anterior synechiae.
i. Neovascular glaucoma
ii. Epithelial downgrowth
iii. Fibrous ingrowth
iv. Flat AC
v. Inflammation

b) Posterior 'pushing mechanism'

The iris is pushed forward by some pathology in the posterior segment.

$\begin{aligned} \text { i. } & \text { Ciliary block glaucoma } \\ \text { ii. } & \text { Intraocular tumours } \\ \text { iii. } & \text { Nanophthalmos } \\ \text { iv. } & \text { Suprachoroidal haemorrhage } \\ \text { v. } & \text { Intravitreal air injection } \\ \text { vi. } & \text { Retrolental fibroplasias }\end{aligned}$

Classification of Developmental glaucoma:

\section{Primary congenital (infantile) glaucoma}

a. Congenital glaucoma

b. Autosomal dominant juvenile glaucoma

c. Glaucoma associated with systemic abnormalities

d. Glaucoma associated with ocular abnormalities

\section{Secondary glaucoma}
a. Traumatic glaucoma
b. Glaucoma with intraocular neoplasm
c. Uveitic glaucoma
d. Lens-induced glaucoma
e. Glaucoma after congenital cataract surgery
f. Steroid induced glaucoma
g. Neovascular glaucoma
h. Secondary angle-closure glaucoma
i. Glaucoma with elevated episcleral pressure
j. Glaucoma secondary to intraocular infection

Specific diseases have been sub-classified, such as POAG types, based on various appearances of the damaged optic nerve ${ }^{19}$, or classification of disease stages by visual field damage ${ }^{20}$; or the angle closure glaucomas based on IOP levels and gonioscopic configurations as correlated with ultrasonic biomicroscopy. ${ }^{21}$

\section{Primary Open Angle Glaucoma}

Primary open angle glaucoma (PAOG) is a chronic progressive optic neuropathy characterised by an open, normal-looking ac and increased intraocular pressure (IOP) without any apparent ocular or systemic abnormality that might account for the raised IOP. Typical optic nerve head damage $(\mathrm{ONH})$ or glaucomatous visual field abnormalities. ${ }^{9}$ Intraocular pressure is the major risk factor and is elevated above the statistical 'normal' range in a majority of cases. It is also the only modifiable risk factor.

\section{Risk factors}

Intraocular pressure: "normal" intraocular pressure may be defined as that pressure which does not lead to glaucomatous damage of the $\mathrm{ONH}$. The commonly used IOP level of $21 \mathrm{~mm}$ hg is based on the observation of two standard deviations (SDS) above the mean of a gaussian distribution curve, and a clinically measured IOP greater than this level is considered elevated. ${ }^{9}$ Evidence from animal studies indicates that elevated IOP can cause glaucomatous ONH changes. ${ }^{22,23}$ Population surveys also corroborate the increase in prevalence of open angle glaucoma with increasing iop. ${ }^{24-26}$ Normal tension glaucoma and ocular hypertension are entities that complete the spectrum of open angle glaucoma disease and have been shown to be associated with a greater severity of disease with intraocular pressures on the higher side in control studies. ${ }^{27-29}$

Age of the patient: The prevalence increases with age. Age is also a risk factor for the conversion from ocular hypertension to open-angle glaucoma. $^{24,25,30-32}$

Sex: males have a higher prevalence of poag. ${ }^{33,34}$

Race: African descent is known to be a risk factor for the development of POAG. ${ }^{30}$

Refractive error: Myopia is a risk factor. ${ }^{35,36}$

Corneal thickness: Apart from causing erroneous low reading with applanation tonometry, a risk factor for the conversion of ocular hypertension to POAG, thin corneas may also be, independently, a direct marker for increased vulnerability of the optic nerve. ${ }^{37}$

Systemic factors: Diabetes mellitus, thyroid eye disease, systemic hypertension, cardiovascular disease, peripheral vasospasm, migraine, sleep apnoea and systemic causes of low perfusion pressure have all been suggested as possible causes. ${ }^{1}$

\section{Clinical features}

Optic nerve head: The appearance of the $\mathrm{ONH}$ and peripapillary retina is the single most 
important clinical feature in establishing glaucomatous damage.

Visual abnormalities: Central visual acuity typically remains normal until there is marked visual field loss. Preliminary evidence, on humphrey visual field analyser perimetry suggests that more subtle measures of visual dysfunction, such as contrast sensitivity, colour vision, and motion perception are early indicators of visual dysfunction before the typical visual field loss develops. ${ }^{9}$ In addition to those with consistently elevated IOP, there are individuals who exhibit optic nerve features suggestive of early glaucoma or who have suspicious field defects. To include these categories and identify a subpopulation of individuals or eyes at increased risk for POAG, the term glaucoma suspect was advocated by Shaffer. ${ }^{9}$

\section{Definition of glaucoma suspect ${ }^{38}$}

Open angle by gonioscopy and one of the following in atleast one eye:

- IOP consistently > $21 \mathrm{~mm}$ hg by applanation tonometry

- Appearance of the optic disc or RNFL suggestive of glaucomatous damage

- Abnormalities of the nerve fibre layer especially at the superior or inferior poles

- Disc haemorrhages

- Asymmetric appearance of the disc or rim between fellow eyes (e.g. Cup-to-disc ratio difference $>0.2$ ) suggesting loss of neural tissue

- Visual fields suspicious for early damage

Ocular hypertension: Patients with an 'elevated' IOP but without the signs of glaucomatous damage in the $\mathrm{ONH}$ or any visual defects are referred to as having ocular hypertension. ${ }^{39}$

Normal tension glaucoma: A clinical subset of PAOG, it has similar disc and field changes but pressures remain in the statistical 'normal' range without treatment. ${ }^{9}$

\section{Angle closure glaucoma:}

Angle closure is defined as the opposition of the peripheral iris against the $\mathrm{tm}$, resulting in obstruction of aqueous outflow. The presence of angle closure with evident optic nerve damage is termed angle closure glaucoma. ${ }^{9}$ On the basis of signs and symptoms and the time course of the disease three types can be distinguished:

I) Acute angle-closure,

Ii) Sub acute angle-closure glaucoma and

Iii) Chronic angle-closure glaucoma. ${ }^{40}$
This classification is however, an oversimplification, as it neither depicts the natural history of disease progression, nor does it contribute to stage-specific management and interventions. The new classification takes into account the assessment of IOP, gonioscopy, and disc and visual field evaluation. Therefore, it depends on the presenting patients' clinical profile rather than the time course and symptom history for staging.

1) Primary angle closure suspect (PACS): greater than $270^{\circ}$ of irido-trabecular contact plus absence of peripheral anterior synechiae (pas) plus 'normal IOP', disc, and visual field.

2) Primary angle closure (PAC): greater than $270^{\circ}$ of irido-trabecular contact with elevated IOP and/or pas plus normal disc and visual field examinations.

3) Primary angle-closure glaucoma (PACG): greater than $270^{\circ}$ of irido-trabecular contact plus elevated IOP plus optic nerve and visual field damage.

Acute primary angle closure remains a specific observable presentation of the disease, requiring emergent intervention. ${ }^{1}$ acute angle-closure glaucoma is characterised by severe pain in the distribution of the trigeminal nerve, redness and blurred vision. On examination, there is conjunctival hyperaemia, cloudy cornea and a middilated fixed pupil. There is marked IOP elevation in the range of $40 \mathrm{~mm} \mathrm{hg}$ to greater than $60 \mathrm{~mm}$ $\mathrm{hg}$, with a severe reduction in central visual acuity. Sub acute angle-closure glaucoma is intermittent, self limited, IOP elevations accompanied by prodromal symptoms of headache, haloes and blurred vision but with normal IOP in the interparoxysmal period, in patients with an occludable angle. Chronic angle-closure glaucoma is typically asymptomatic until advanced visual field loss. Patients present with occludable angles having pas of more than 180 degrees and a chronically elevated IOP. ${ }^{41,42}$

\section{Risk factors:}

Primary angle-closure mechanisms can present with three site-specific disturbances in the anterior segment i.e. the pupillary block mechanism, ciliary body anomalies (e.g. Plateau iris syndrome) and lens induced. Demographic risk factors are as follows:

1) Age of the patient: Incidence increases with age. Age associated changes cause an 
increased tendency towards pupillary block, (especially $6^{\text {th }}-7^{\text {th }}$ decades).. However, it can present at any age. ${ }^{43,44}$

2) Sex: Female predominance (2-3 times) possibly because of shallower ACS. ${ }^{45}$

3) Heredity of the patient: Because configuration of the ac is under polygenic influence. Inuit population have the highest known prevalence. $^{46,47}$

4) Refractive error: smaller dimensions of depth and volume of ac predispose hyperopes to primary angle-closure glaucoma. ${ }^{45,48}$

Ocular risk factors and mechanisms: these include reduced axial ac depth and volume, short axial length, steeper radii of corneal curvature, and thicker lens in particular. ${ }^{49,50}$ Based on an increased iop $\geq 21 \mathrm{~mm} \mathrm{hg}$ on two or more occasions and/or optic disc changes suggestive of glaucoma, patients should undergo a comprehensive evaluation using slit lamp, fundoscopy, gonioscopy, applanation tonometry and perimetry to determine and classify the various subtypes of glaucoma presenting in clinic. The risk factors, clinical history, signs, symptoms and clinical profile of each patient should be recorded. $^{51}$

\section{CONCLUSION}

One of the leading causes of blindness is glaucoma. It can be prevented by early diagnosis and treatment. Patients with a family history of the disease should be referred by the primary care physicians for a complete ophthalmologic examination. Many severity classification methods have been proposed, although none have had widespread use in clinical practice. Further, assessment of the optic nerve by direct ophthalmoscopy may rule out suspicious signs of optic nerve damage so that prompt referral to an eye care specialist can be done.

\section{REFERENCES}

1. Stamper RL, Lieberman MF, Drake MV. Becker-shaffer's diagnosis and therapy of the glaucomas. $8^{\text {th }}$ ed. St.louis: mosby; 2009.

2. Van Buskrirk EM, Cioffi CA. Glaucomatous optic neuropathy. American journal of ophthalmology 1992;113(4):447-452.

3. Quigley HA. Neuronal death in glaucoma. Prog retin eye res 1999; 18:39-57.
4. Hitchings RA, Spaeth GL. The optic disc in glaucoma. I: classification. British journal of ophthalmology 1976;60(11):778-85.

5. Mikelberg FS, Drance SM. The mode of progression of visual field defects in glaucoma. American journal of ophthalmology 1984;98(4):443.

6. Kitazawa Y, Yamamoto T. Glaucomatous visual field defects: their characteristics and how to detect them. Clin neurosci 1997;4:279.

7. Leske MC, Podgor MJ. Intraocular pressure, cardiovascular risk variables, and visual field defects. American journal of epidemiology 1983; 118(2):280-7.

8. Quigley HA, Enger C, Katz J, Sommer A, Scott R, Gilbert D. Risk factors for the development of glaucomatous field loss in ocular hypertension. Archives of ophthalmology 1994;112(5):644-9.

9. Shields MB. Shields' textbook of glaucoma. $6^{\text {th }}$ ed. Philadelphia: williams \& wilkins; 2011.

10. Weih LM et al prevalence and predictors of open angle glaucoma: results from the visual impairment project, ophthalmology 2001;108:1966.

11. Shields MB, Ritch R, Krupin T: classifications of the glaucomas. In: ritch $r$, shields $\mathrm{mb}$, krupin $t$, editors: the glaucomas, $2^{\text {nd }} e d n$., st.louis, mosby, 1996.

12. Quigley HA: number of people with glaucoma worldwide, br j ophthalmol 1996;80:389.

13. Thylefors B, Negrel AD: the global impact of glaucoma, bull worth health organ 1994;72:323.

14. Quigley H, Broman A: number of people with glaucoma worldwide in 2010 and 2020, br j ophthalmol 2006;90:262.

15. Johnson G, Quigley $H$ : the glaucomas. In: johnson $\mathrm{g}$, minassian $\mathrm{d}$, weale $\mathrm{r}$, west $\mathrm{s}$, editors: the epidemiology of eye disease, $2^{\text {nd }}$ edn., london, arnold, 2003. Pp 222-239.

16. Foster P, He M, Liebmann J: epidemiology, classification and mechanism. In: weinreb rn, editor: angle closure and angle closure glaucoma, the hague, kugler, 2006. Pp 1-20.

17. Mardin CY, Hothorn T, Peters A, Junemann AG, Nquyen NX, Lausen B. J glaucoma.2003 aug;12(4):340-6.

18. Quigley HA, Congdon NG, Friedman DS: glaucoma in china (and worldwide): changes in established thinking will decrease 
preventable blindness, br $\mathrm{j}$ ophthalmol 2001;85:1271.

19. Spaeth GL: a new classification of glaucoma including focal glaucoma, surv ophthalmol 1994;38:s9.

20. Mills RP, Budenz DL, Lee PP et al: categorizing the stage of glaucoma from prediagnosis to end-stage disease, am j ophthalmol 2006;141:24-30.

21. Kim YY, Jung HR: clarifying the nomenclature for primary angle-closure glaucoma, surv ophthalmol 1997;42:125.

22. Gaasterland DE, Tanashima T, Kuwabara T: axoplasmic flow during chronic experimental glaucoma. I. Light and electron microscopic studies of the monkey optic nerve-head during development of glaucomatous cupping. Invest ophthalmol vis sci 1978;17:838.

23. Quigley HA, addicks em: chronic experimental glaucoma in primates. Ii. Effect of extended intraocular pressure elevation on optic nerve head and axonal transport. Invest ophthalmol vis sci 1980;19:137.

24. Hollows FC, graham pa: intraocular pressure, glaucoma and glaucoma suspects in a defined population, br j ophthalmol 1966;50:570.

25. Bengtsson B: the prevalence of glaucoma. $\mathrm{Br} \mathrm{j}$ ophthalmol 1981;65:46.

26. $\mathrm{Hu} \mathrm{Z}$ et al. An epidemiologic investigation of glaucoma in beijing city and shun-yo county. Chin j ophthalmol. 1989;25:115.

27. Cartwright MJ, Anderson DR: correlation of asymmetric damage with asymmetric intraocular pressure in normal-tension glaucoma (low-tension glaucoma), arch ophthalmol 1988;106:898.

28. Crichton A et al: unequal intraocular pressure and its relation to asymmetric visual field defects in low-tension glaucoma, ophthalmology 1989;96:1312.

29. Gordon MQ, et al: the ocular hypertension treatment study: baseline factors that predict the onset of primary open-angle glaucoma: arch ophthalmol 2002;120:714.

30. Tielsch JM et al: racial variations in the prevalence of primary open angle glaucoma. The baltimore eye survey, jama 1991;266:369.

31. Klein BE et al: prevalence of glaucoma. The beaver dam eye study, ophthalmology 1992;99:1499.
32. Leibowitz HM et al: the framingham eye study: monograph, surv ophthalmol 2002;120:714.

33. Kahn ha, milton rc: alternative definitions of open-angle glaucoma: effect on prevalence and associations in the framingham eye study, arch ophthalmol 1980;98:2172.

34. Leske MC et al: the barbados eye study: prevalence of open-angle glaucoma, arch ophthalmol 1994;112:82.

35. Mastropasqua $L$ et al: prevalence of myopia in open angle glaucoma, eur $\mathrm{j}$ ophthalmol 1992;2:33.

36. Tomlinson A. Phillips CI: applanation tension and axial length of the eyeball, br $\mathrm{j}$ ophthalmol 1970;54:548.

37. Brandt JD: corneal thickness in glaucoma screening, diagnosis and management, curr opin ophthalmol 2004;15:85.

38. American academy of ophthalmology. Primary open-angle glaucoma suspect, preferred practice pattern, san francisco, ca: american academy of ophthalmology, 2005. Available at: http://www.aao.org/ppp. Accessed june 10, 2010.

39. European glaucoma society. Terminology and guidelines for glaucoma, $2^{\text {nd }}$ ed. Savona; italy: dogma s.r.l., 2003.

40. Clemmesen V. Problems in gonioscopic screenings in greenland: technique, classification of findings and diagnosis. Acta ophthalmol 1971; 49: 59-64.

41. Sood NN, Jain RC, Agarwal HC. Ocular biometry in primary angle closure glaucoma in indians. Indian j med res 1988; 88:190-1.

42. Sihota R, Lakshmaiah NC, Agarwal HC, Pandey RM, Titiyal JS. Ocular parameters in the subgroups of angle closure glaucoma. Clin exp ophthalmol 2000; 28:253-8.

43. Lehrfeld L, Reber J: glaucoma at the wills hospital, arch ophthalmol 1937;18:712.

44. Lowe RF. angle-closure glaucoma: acute and sub acute attacks: clinical types, trans ophthalmol soc aust 1961;21:65.

45. Fontana ST, Brubaker RF: volume and depth of the anterior chamber in the normal aging human eye, arch ophthalmol 1980;98:1803.

46. Probert LA: a survey of hereditary glaucoma, can med assoc j 1952;66:563.

47. Biro I: notes upon the question of hereditary glaucoma, ophthalmologica 1951;122:228. 
48. Wojciechowski R et al: age, gender, biometry, refractive error, and the anterior chamber angle among alaskan eskimos, ophthalmology 2003;110:365.

49. Quigley HA, Friedman DS, Congdon NG: possible mechanisms of primary angle-closure and malignant glaucoma, $\mathrm{j}$ glaucoma 2003; 12:167.

Source of support: Nil

Conflict of interest: None declared
50. Foster P, He M, Liebmann J: epidemiology, classification and mechanism. In: weinreb rn, friedman ds, editors: angle closure and angle closure glaucoma, the hague, kugler, 2006.

51. Hitchings RA. Intraocular pressure and circulation at the disc in glaucoma. Acta ophthamologica scandinavia supplement. 1997; 220: 15-20; 20-2.

This work is licensed under CC BY: Creative Commons Attribution 4.0 License. 